\title{
Impacto de los precios de los metales en la estructura de capital de las empresas minero-metalúrgicas en América Latina (2004-2014)
}

\author{
The impact of metals' prices on the capital structure of mining and \\ metallurgic firms in Latin America (2004-2014)
}

\author{
Roberto J. Santillán Salgado ${ }^{a,{ }^{*}}$, Alejandro Fonseca Ramírez ${ }^{\mathrm{a}}$ y \\ Francisco Venegas Martínez \\ ${ }^{a}$ EGADE-ITESM, Monterrey, México \\ ${ }^{b}$ Instituto Politécnico Nacional, México
}

Recibido el 22 de septiembre de 2015; aceptado el 19 de enero de 2016

Disponible en Internet el 27 de junio del 2018

\section{Resumen}

Este trabajo examina los efectos de corto y mediano plazo de las fluctuaciones de los precios internacionales de varios productos minerales (oro, plata, cobre, zinc) y metalúrgicos (aluminio, acero) sobre la estructura de capital de las empresas de la industria minera y metalúrgica listadas en los mercados bursátiles de Argentina, Brasil, México y Perú. Se utilizan datos trimestrales para el periodo 2000-2014. Consistente con la teoría del Orden Selectivo de Preferencia Dinámico "Dynamic Pecking Order", los resultados del análisis econométrico confirman que la rentabilidad y los precios de los productos se relacionan negativamente con en el apalancamiento financiero de las empresas.

Códigos JEL: G13, G32, G33.

Palabras clave: Precio de productos minerales; Estructura de capital; Desempeño corporativo.

\footnotetext{
*Autor para correspondencia.

Correo electrónico: roberto.santillan@itesm.mx (R.J. Santillán Salgado).

La revisión por pares es responsabilidad de la Universidad Nacional Autónoma de México.
} 


\begin{abstract}
This work examines the short and medium-term effects of the international prices of several mineral (gold, silver copper, zinc) and metallurgic products (aluminum, steel), on the capital structure of mining and metallurgic companies listed in the stock markets of Argentina, Brazil, Mexico and Peru. The study uses quarterly data for the period 2000-2014. Consistent with the Dynamic Pecking Order theory, the findings confirm that profitability and the price of the products relate negatively with the firms' financial leverage.
\end{abstract}

JEL classification: G13, G32, G33.

Keywords: Mineral products’ prices; Capital Structure; Corporate Performance.

\title{
Introducción
}

La estructura de capital de una empresa consiste en la proporción de deuda y recursos propios (acciones comunes y preferentes) que utiliza para financiar sus inversiones. La teoría de la determinación de la Estructura de Capital se ocupa de analizar la manera como la empresa toma esa decisión y, en consecuencia, divide los flujos de efectivo generados por su operación normal entre: a) un componente fijo, destinado a pagar las obligaciones de la empresa frente a sus acreedores; y b) un componente residual que corresponde a los accionistas (comunes y preferentes). Esa decisión tiene una connotación estratégica de la mayor importancia debido a que el nivel de apalancamiento determina la sensibilidad del flujo libre de efectivo y, por lo tanto, del valor de la empresa, a los riesgos del mercado. Por esa razón, es un tema que recibe gran atención en la investigación financiera.

El objetivo de este estudio es determinar de qué manera se presenta la relación entre las fluctuaciones de los precios de los principales metales preciosos y de uso industrial (oro, plata, aluminio, cobre, zinc y acero), y la rentabilidad y estructura de capital de una muestra de productoras mineras y metalúrgicas cuyas acciones forman parte de los índices bursátiles de los mercados de Argentina, Brasil, México y Perú.

En un primer momento se recurre a la metodología de Análisis de Factores para reducir la dimensionalidad de las variables explicativas (precios de los commodities) del modelo y, en seguida, se lleva a cabo un Análisis de Regresión con datos de sección cruzada y series de tiempo combinados (Transversal), se realizan pruebas para determinar la oportunidad de agregar efectos fijos o efectos aleatorios en la estimación, y se concluye que la regresión transversal es suficiente para explicar satisfactoriamente el modelo. Las conclusiones principales se apoyan en la evidencia descubierta para sugerir medidas de administración de riesgo para las empresas de la industria minera y metalúrgica, así como para recomendar a los administradores de portafolios de acciones y bonos emitidos por las mismas tomar en cuenta la dinámica observada por su estructura de capital.

Los resultados obtenidos a partir de nuestro análisis corroboran las predicciones de la Teoría del Orden Selectivo de Preferencia Dinámico "Dynamic Pecking Order", en el sentido de que los niveles de apalancamiento de las empresas son sensibles a su capacidad de generar recursos internos. En la segunda parte se revisan los antecedentes de la Teoría de la Estructura de Capital. En la tercera, se revisa la evidencia arrojada por estudios diversos sobre los determinantes de 
la Estructura de Capital. En la cuarta parte, se revisan trabajos sobre estructura de capital en América Latina y, especialmente, en el sector minero-metalúrgico. En la quinta, se analiza la evolución reciente de los precios de los productos estudiados, y se presenta la muestra de empresas minero-metalúrgicas listadas en los índices de Argentina, Brasil, México y Perú. En la sexta parte, se abordan los aspectos metodológicos del análisis, se presentan los resultados y se interpreta su significado. Finalmente, se concluye con algunas reflexiones y recomendaciones.

\section{La teoría clásica de la estructura de capital}

El trabajo seminal de Miller y Modigliani (1958) constituye un punto de partida de la mayor importancia para el desarrollo de la teoría moderna de la estructura de capital en las empresas.

La contribución seminal de estos autores a la teoría que busca una explicación de cómo se determina la Estructura de Capital en las empresas conduce a varias conclusiones de la mayor importancia. En su primera versión, en la cual se asume la inexistencia de los impuestos y de los costos de bancarrota, resulta imposible determinar una estrategia financiera idónea ya que, independientemente de las proporciones correspondientes a la deuda y al capital, el costo de capital promedio ponderado se mantiene constante. Sin embargo, al reconocer la existencia de impuestos, pero bajo el supuesto de que no existen los costos de bancarrota, se puede proponer una estrategia financiera que se beneficia al máximo del escudo fiscal y potencia la rentabilidad de la empresa ${ }^{2}$ para sus accionistas y que conduce a utilizar el máximo posible de endeudamiento ${ }^{3}$ aunque, obviamente, esta versión está alejada del mundo real (Miller y Modigliani, 1963).

El desarrollo de estas ideas evolucionó rápidamente durante los años siguientes y, en 1973, Litzenberg (1973) propuso por vez primera en la teoría conocida como "Costo de Oportunidad de las Decisiones de Financiamiento" en la cual se afirma que una empresa sigue una estrategias de selección de fuentes de financiamiento con base el análisis costo-beneficio que resulta de comparar los beneficios de la utilización de deuda, con el costo relacionado a la posibilidad de bancarrota.

Para poner en justa perspectiva la propuesta anterior es necesario hacer mención a otros trabajos. Una de las contribuciones más importantes para el desarrollo de la teoría fue el trabajo de Jensen y Meckling (1976), quienes plantearon que los incentivos de los administradores en una empresa están alineados con el objetivo de maximizar el valor del capital accionario, en lugar del valor total de la empresa. De acuerdo con esa lógica, cuando los administradores disponen de recursos excedentes, se involucran en estrategias que transfieren riesgos a los tenedores de bonos. Al favorecer proyectos riesgosos que benefician a los accionistas en caso de éxito, por muy improbable que éste sea $^{5}$, pero que potencialmente resultarían en pérdidas importantes a los tenedores de bonos en caso de no tener éxito, la empresa lleva a cabo una estrategia conocida como "sobre inversión"

\footnotetext{
${ }^{2}$ Un administrador racional utilizará deuda solamente cuando el costo de la misma es menor a la rentabilidad esperada de las inversiones que se financiarán. Por lo tanto, el excedente por encima del costo de la deuda se acumula al beneficio obtenido por los accionistas.

${ }^{3}$ Bajo este escenario podría asumirse un nivel de apalancamiento del 99.99\%, a fin de que exista al menos un "dueño" que reciba la totalidad de los beneficios.

${ }^{4}$ Traducción libre del término original, en inglés: "Trade-Off Theory".

${ }^{5}$ Los administradores invierten incluso en aquellos proyectos en los cuales no se espera obtener un valor presente neto positivo, al menos que se presenten condiciones extraordinarias.

${ }^{6}$ En inglés, el término utilizado es "overinvestment".
} 
del "problema de agencia" que resulta de la conducta de los administradores, demandarán un premio adicional por riesgo cuando la empresa pretenda emitir deuda adicional.

Myers (1977) desarrolló una argumentación similar para explicar el problema conocido como "sub-inversión" ". Su argumento es que los administradores tienen un incentivo para no invertir en proyectos nuevos aun cuando su valor presente neto es positivo, si las ganancias esperadas beneficien principalmente a los tenedores de bonos y sólo marginalmente a los accionistas.

Myers (1984) cuestionó que la estructura óptima de capital puede explicarse a partir solamente del análisis de los costos y los beneficios de utilizar deuda ${ }^{8}$ en un contexto de asimetría de información entre los accionistas y los gerentes de la empresa, y sugirió que el Orden Selectivo de Preferencia de las Fuentes de Financiamiento no debe concebirse como algo estático. De no existir costos de ajuste, la estructura de capital observada en las empresas debería ser la óptima en todo momento. No obstante, debido a que sí existen, normalmente existen rezagos en el ajuste del apalancamiento hacia el nivel óptimo. Las empresas no pueden compensar inmediatamente los efectos de acontecimientos que las desvían del nivel óptimo, por lo que es de esperar (como sucede en la realidad) que exista una dispersión transversal entre la razón de apalancamiento efectiva en una muestra de empresas que tienen la misma razón objetivo de palanca financiera. A este enfoque se le conoce como la Teoría del Orden Selectivo de Preferencia Dinámico9.

Myers y Majluff (1984) desarrollaron una propuesta diferente sobre los determinantes de la estructura de capital en las empresas. Estos autores argumentaron que existe asimetría en la información que tienen los empleados de la empresa ("insiders"), y la que tienen los agentes económicos externos a la empresa ("outsiders", que incluye a inversionistas y analistas del mercado). Algunas de las implicaciones teóricas son que: a) los administradores, poseedores de una información superior sobre el valor real de la empresa, no emitirán nuevas acciones cuando consideren que la empresa se encuentra subvaluada porque hacerlo lleva a una dilución de las acciones de los actuales accionistas; b) los administradores emitirán acciones solamente cuando consideren que las acciones se encuentran sobrevaluadas en el mercado y, en ese sentido, el anuncio de una nueva emisión actúa como una señal que es interpretada por el mercado en el sentido de que las acciones están muy caras, ocasionando una reacción a la baja en el precio.

Así, la mejor estrategia de financiamiento consiste en utilizar los fondos generados internamente, siempre que estén disponibles, lo cual evita los problemas de asimetría de información. Si los fondos generados por la operación no son suficientes para cubrir las necesidades de operación, inversión y crecimiento, las empresas emitirán deuda, pues al ser un compromiso contractual constante en su valor (al menos desde la perspectiva del inversionista que la mantiene hasta vencimiento), se ve menos afectada por la asimetría de información que el valor de las acciones.

De los argumentos anteriores puede concluirse que la determinación de cuál debe ser la estructura óptima de financiamiento de una empresa requiere evaluar cuidadosamente los costos de agencia asociados a la utilización de financiamiento externo (transferencia de riesgos y subinversión), ponderándolos contra los costos de agencia relacionados con exceso de efectivo y ausencia de compromisos financieros (el problema del flujo libre de efectivo). Pero, una vez determinada la proporción óptima de deuda con respecto al capital, la empresa no tendrá más alternativa que la búsqueda consistente de ese nivel.

\footnotetext{
${ }^{7}$ En inglés, el término utilizado es "underinvestment".

${ }^{8}$ Teoría de la Elección de la Estructura de Capital con Base en el Costo de Oportunidad, o Trade-Off Theory.

${ }^{9}$ Traducción del nombre técnico en inglés "Dynamic Pecking Order Theory".
} 


\section{Los factores que determinan la estructura de capital}

La literatura que lleva a cabo una contrastación empírica de los determinantes de la estructura de capital de las empresas es muy amplia. Sin embargo, aún existe ambigüedad sobre cuál de las distintas posiciones teóricas existentes explica mejor el fenómeno y, por lo tanto, es un campo en el cual se requiere más trabajo de investigación. Los notables trabajos de revisión de la literatura realizados por Harris y Raviv (1991), o Graham y Lear (2013) discuten múltiples trabajos sobre el tema, pero difícilmente comprenden la mayoría de las propuestas teóricas, ni dan cuenta de los resultados de todos los estudios de contrastación empírica pertinentes al tema. Pero, aunque indudablemente los trabajos identificados por estos autores (y muchos otros) son cada vez más abundantes y variados, la teoría sobre los determinantes de la estructura de capital se encuentra en una etapa de definiciones y es muy necesario un mayor esfuerzo de investigación en esta línea.

Harris y Raviv (1991) presentan una amplia revisión de la literatura existente hasta fines de los años 1980s sobre las teorías que explican la estructura de capital. La revisión incluye artículos académicos que explican el fenómeno a partir de los costos de agencia, la asimetría de información, las interacciones entre mercados de productos y/o mercados de insumos, así como otras consideraciones relacionadas con el control corporativo. Se trata de un trabajo que revisa investigaciones en las cuales se desarrollan distintos modelos teóricos y estudios empíricos cuyo propósito es cotejar los modelos con la realidad. La contribución más importantes de este es la identificación de un conjunto de variables cuya relación con la estructura de capital hace de ellas candidatas ideales para llevar a cabo la contrastación de la teoría con la realidad y las regularidades o hechos estilizados que se encuentran ampliamente documentados.

Graham y Lear (2013) presentan una revisión detallada de los trabajos de investigación empírica sobre estructura de capital publicados desde el año 2005 y hasta los primeros años del segundo decenio del siglo XXI. Para establecer un orden en el análisis, clasifican los trabajos como pertenecientes a una de tres perspectivas que explican las variaciones en la estructura de capital: a) entre empresas; b) entre industrias; $y, c)$ de una misma empresa a través del tiempo. También evalúan la capacidad explicativa de los enfoques del Costo de Oportunidad de las Fuentes de Financiamiento y del Orden Selectivo de Preferencia Dinámico de las Fuentes de Financiamiento, y destacan cuáles son sus limitaciones. Analizan trabajos sobre la manera como la oferta de capital afecta el nivel de apalancamiento, así como algunos aspectos puntuales de los contratos de crédito que no habían sido investigados con minuciosidad anteriormente, y se preocupan por comprender porqué los cambios en la estructura de capital de las empresas tienen poca influencia sobre su valor en un rango amplio de niveles de apalancamiento, porqué las estimaciones de la velocidad con la cual tienen lugar los cambios de estructura de capital están sesgadas, y otros muchos aspectos sobre la estructura de capital de las empresas no tomados en consideración anteriormente.

Otros dos trabajos que es oportuno mencionar en esta sección son el de Frank y Goyal (2009) y el de Bessler, Drobetz y Kazemieh (2011), porque apuntalan el marco de referencia de esta investigación y, específicamente, sirven para justificar la elección de los precios de los commodities como variable explicativa de los niveles de apalancamiento de las empresas.

De acuerdo con Frank y Goyal (2009), la teoría del Orden Selectivo de Preferencias es una de las más influyentes sobre la estructura de capital. Debido al problema de selección adversa, las empresas prefieren financiarse internamente pero, cuando se requiere financiamiento externo, 
prefieren deuda por encima de capital debido a los menores costos de información asociados con la emisión de bonos (Myers 1984). Por lo tanto, si una empresa determina su estructura de capital de acuerdo con lo previsto por la Teoría del Orden Selectivo de Preferencias, en una regresión del nivel de apalancamiento contra el déficit financiero, debería observarse un coeficiente de pendiente igual a uno. Por ejemplo, Shyam-Sunder y Myers (1999) encuentran un resultado favorable para esa predicción en una muestra de 157 empresas, entre 1971 y 1989. Es decir, la evidencia avala estadísticamente la validez de la Teoría del Orden Selectivo de Preferencias Dinámico.

En contraste, Bessler, Drobetz y Kazemieh (2011) afirman que tanto las variables que representan el apalancamiento en las empresas como aquellas que frecuentemente se asumen como sus determinantes, deben estar relacionadas con las teorías que explican la naturaleza y funcionamiento de la estructura de capital. Esto es relevante debido a que se asume que dichas variables son mediciones indirectas de las fuerzas económicas descritas en la teoría tales como, por ejemplo, los costos asociados al estrés financiero o la asimetría de información. No obstante, el signo esperado de la relación es, a veces, ambiguo, y de ahí la importancia de clarificar en qué casos se cuenta con un consenso respecto al signo de esas relaciones. Tal definición resulta indispensable, por ejemplo, para anticipar los niveles de apalancamiento con base en otras características o factores de entorno de las empresas (como los precios de sus productos).

Una conclusión preliminar de la discusión sobre los determinantes de la estructura de capital es que aún se trata de un capítulo inconcluso en Economía Financiera, y que la nueva evidencia continuamente presentada en la literatura sobre las principales posturas brevemente esbozadas aquí contribuye a sentar las bases para una futura definición categórica a favor de una u otra posición teórica.

\section{Estudios sobre estructura de capital en América Latina}

El estudio de la estructura de capital en los mercados emergentes ha producido notables resultados, por lo que mencionamos solamente dos de los trabajos más destacados como referencia para el lector interesado, pero no desarrollamos el tema con mayor detalle por razones de espacio. Los dos estudios recomendados sobre este tema son Booth, Aivazian, DemirgucKunt y Maksimovic (2001) y Jõeveer (2006).

En el ámbito latinoamericano, la tendencia a producir investigaciones sobre los determinantes de la estructura de capital de las empresas ha tenido un repunte en años recientes e, igual que en el caso de la literatura sobre mercados emergentes, debemos limitar nuestra revisión de las mismas a la mención de aquellas que consideramos más representativas.

El trabajo de Medina, Salinas, Ochoa y Molina (2012), presenta una revisión de estudios empíricos sobre la estructura de capital de empresas colombianas (entre otros), y corrobora la vigencia empírica de la teoría del orden selectivo de preferencias dinámico para empresas colombiano. Mongrut et al. (2010), estudian cómo determinan su política de endeudamiento las empresas corporativas bursátiles de Argentina, Brasil, Chile, México y Perú en el periodo 1995-2007 y, con base en los resultados de un análisis de panel, encuentran evidencia para rechazar la hipótesis de la teoría del Orden de Preferencia Dinámico. Maqueira, Olavarrieta y Zutta (2007) analizan los determinantes de la estructura de capital para una muestra de 113 empresas chilenas, para el periodo de 1990 a 1998. Estos autores contrastan los resultados 
de un modelo Lisrel (Linear Structural Relations) con respecto a los resultados obtenidos por minimos cuadrados ordinarios (MCO) y encuentran diferencias importantes. La lista de trabajos sobre estructura de capital en América Latina crece continuamente y aporta cada vez más resultados de interés para la discusión teórica.

Puesto que este trabajo estudia la estructura de capital en una muestra de empresas minerometalúrgicas de América Latina, vale la pena destacar dos trabajos cuya atención se concentra, precisamente, en empresas de ese sector en la región. Se identificaron dos trabajos que cumplen con el criterio anterior, a saber, el de Paredes, Ángeles y Flores (2015), y el de Paredes y Flores (2012).

Paredes, Ángeles y Flores (2015) utilizan análisis de panel estático y dinámico para explorar los determinantes del apalancamiento de 14 empresas mineras en México, Colombia, Chile, Brasil y Perú, entre el primer trimestre de 2004 y el tercer trimestre de 2014, y contrastan sus resultados con las teorías relevantes. En él se reporta que, en tanto más tangibles son los activos, se observa un mayor nivel de apalancamiento, mientras que la tasa de crecimiento de las ventas y el tamaño de la empresa no tienen un efecto significativo sobre dicha variable. Otro hallazgo es que la rentabilidad reduce el apalancamiento, lo cual es consistente con la teoría del Orden Selectivo de Preferencias Dinámicas, y que los valores rezagados del apalancamiento son más robustos para explicar el valor actual del apalancamiento que las demás variables explicativas.

Paredes y Flores (2012), proponen un análisis de panel para estudiar el comportamiento de la estructura de capital de diez empresas del sector siderúrgico mexicano durante el periodo 2001 -2011. El objetivo de la investigación consiste en determinar la significancia de los factores que inciden en el nivel de apalancamiento, con base en los conceptos de la teoría de la estructura de capital. Estos autores utilizan información financiera de las empresas y una variable macroeconómica, como variable de control. Las variables financieras incluyen el nivel de apalancamiento, el flujo de caja relativo al valor del activo, la tasa de crecimiento de los ingresos, la proporción de activos productivos, y el tamaño de la empresa. La variable macroeconómica es la tasa de crecimiento anual del Producto Interno Bruto. Los resultados del estudio indican que existe una relación muy importante entre el crecimiento de la empresa y los flujos de efectivo que se destinan a las inversiones, por una parte, y el nivel de apalancamiento, por otra. Al aumentar la rentabilidad de la empresa, el flujo de efectivo y los excedentes se pueden invertir en activos productivos, y al mismo tiempo disminuye el nivel de apalancamiento. El modelo de efectos fijos utilizado permite concluir que la relación entre las variables seleccionadas y el nivel de apalancamiento es distinta para cada empresa.Los resultados son consistentes con el Orden Selectivo de Preferencias.

Es muy probable que tanto la teoría del Costo de Oportunidad de las Decisiones de Financiamiento como la teoría del Orden Selectivo de Preferencia sean, aún combinadas, explicaciones aún parciales de un fenómeno más complejo. La incidencia de conflictos de agencia, volatilidad de la industria, diferenciación del producto, y otros factores, dejan en claro que, si bien la teoría tradicional explica una buena parte de los hechos estilizados, tal como lo dejan establecido Frank y Goyal (2003), existen muchas variables que pueden influir en los niveles de apalancamiento de las empresas.

\section{Los precios de los metales mineros y metalúrgicos, y las empresas latinoamericanas que los producen}

Puesto que en condiciones normales, los cambios tecnológicos en la minería son muy graduales y, por lo tanto, los costos de operación de las empresas de la industria son muy estables en el tiempo, es posible suponer que las fluctuaciones de los precios de los minerales, sujetos 
a frecuentes altibajos, relacionados generalmente con el ciclo económico, pero en ocasiones también a situaciones geopolíticas e incluso decisiones estratégicas de los grandes productores mundiales, son un factor muy importante en la determinación de los niveles de rentabilidad y la generación de flujo operativo de las empresas mineras. En tal sentido, confirmar si existe incidencia del precio de los minerales sobre la estructura de capital de las empresas mineras se enmarca dentro de la teoría del Orden Selectivo de Preferencias. Cuando los precios de los minerales son elevados, la mayor utilidad y generación de flujo operativo, reducen los requerimientos de financiamiento externo, y su efecto debe reflejarse en la estructura de capital.

La teoría financiera sugiere que el nivel de apalancamiento de las empresas debería medirse como la razón de deuda a capital, ambos expresados a su valor de mercado. Sin embargo, la dificultad para obtener información del valor de mercado del pasivo total obliga a calcularlo utilizando el valor en libros del pasivo, combinado con el valor de mercado del capital. Así, el numerador de esta razón es un valor contable proveniente del balance general, el cual se modifica solo gradualmente en el tiempo; pero el denominador cambia continuamente, en función del cambio de precio de las acciones.

En este trabajo se mide el nivel de apalancamiento de las empresas de la muestra en términos del valor contable de la deuda en el numerador y del valor de capitalización (de mercado) de sus acciones, en el denominador, puesto que esa medición del nivel de apalancamiento responde de manera dinámica a las fluctuaciones de los precios de los minerales que producen las empresas de la muestra.

En la revisión de la literatura sobre los determinantes de la estructura de capital de las empresas presentado antes, se refiere que existe un amplio número de trabajos que exploran cuáles son los factores que influyen en los niveles de apalancamiento de las empresas. Así, la teoría del Costo de Oportunidad de las Fuentes de Financiamiento (trade-off) postula que los principales determinantes de la estructura de capital son los beneficios derivados del escudo fiscal de la deuda, versus los costos potenciales de bancarrota; o bien, la teoría del Orden Selectivo de las Fuentes de Financiamiento (Pecking Order Theory) propone que la cantidad relativa de cada una de las fuentes de financiamiento que utilizan las empresas depende del nivel de asimetría de información que existe entre los inversionistas con respecto al conjunto de información que manejan los administradores. Sin embargo, más allá de estas explicaciones fundamentales de los determinantes de la estructura de capital de las empresas, es posible identificar factores tan diversos como, por ejemplo, el grado de diferenciación de los productos, la intensidad de los gastos en publicidad o en investigación y desarrollo, y otros muchos. En particular, en este trabajo se propone modelar la sensibilidad de la razón Deuda Total/Valor de Capitalización de las Acciones a las variaciones de los precios internacionales de los minerales para una muestra de empresas mineras en Latino América.

\section{Comportamiento de los precios de los productos mineros y del acero entre 2004 y 2014}

Durante los once años transcurridos entre enero de 2004 y diciembre de 2014, los precios de la mayoría de los commodities experimentaron variaciones muy importantes. Desde comienzos del siglo y hasta el año 2007 había tenido lugar un crecimiento importante en los mismos, impulsado por la fuerte demanda de materias primas generada como consecuencia de la elevada tasa de crecimiento de la economía china y de otros países emergentes, junto con la estabilidad macroeconómica global que permitió, un crecimiento consistente, aunque lento, de la mayoría de los países industrializados. 
Sin embargo, ante la turbulencia provocada por la Crisis Financiera 2007-2009 cambió radicalmente ese escenario. Los agentes económicos buscaron un refugio seguro, tradicionalmente los metales preciosos y las monedas duras, y así se dio un impulsó importante a la alza al precio del oro (y, en menor medida, la plata), junto con la apreciación del dólar americano frente al resto de las monedas.

Si bien durante los primeros años del siglo la tendencia en los precios de los metales fue consistentemente al alza, consistente con la alta correlación positiva históricamente observada entre sus precios (ver Tabla 1 y Figuras 1 y 2), solamente el oro y la plata se consideran un refugio seguro ante la incertidumbre. Por lo tanto, como consecuencia de las graves repercusiones del colapso financiero mundial sobre la actividad económica, los precios de los metales de uso principalmente industrial siguieron un comportamiento claramente diferenciado con relación a los metales preciosos. No obstante, la correlación entre los precios de los seis metales fue muy alta durante el periodo, siempre por encima de $0.40 \mathrm{y}$ en algunos casos hasta por encima de 0.90 .

Tabla 1.

Correlaciones entre precios de los minerales seleccionados.

\begin{tabular}{|c|c|c|c|c|c|c|}
\hline & Oro & Plata & Cobre & Acero & Aluminio & Zinc \\
\hline Oro & 1.000 & 0.959 & 0.844 & 0.885 & 0.402 & 0.462 \\
\hline Plata & 0.959 & 1.000 & 0.864 & 0.875 & 0.494 & 0.500 \\
\hline Cobre & 0.844 & 0.864 & 1.000 & 0.915 & 0.795 & 0.798 \\
\hline Acero & 0.885 & 0.875 & 0.915 & 1.000 & 0.667 & 0.587 \\
\hline Aluminio & 0.402 & 0.494 & 0.795 & 0.667 & 1.000 & 0.849 \\
\hline Zinc & 0.462 & 0.500 & 0.798 & 0.587 & 0.849 & 1.000 \\
\hline
\end{tabular}

Fuente: Cálculos propios con datos de Standard \& Poors' Capital Markets Data Base.

El oro aumentó desde un precio cercano a los 400 dólares por onza en enero de 2004, hasta un máximo de 1,772 en septiembre de 2012, es decir, un crecimiento porcentual del 343\%, para después registrar un ajuste importante respecto a su nivel máximo, y buscar un nuevo piso alrededor de 1, 200 dólares hacia el final del periodo analizado. Por su parte, el precio de la plata pasó de poco más de 7 dólares la onza, hasta casi 38 dólares entre enero de 2004 y marzo de 2011, es decir un crecimiento de $442 \%$. A partir de ese momento observó un patrón de ajuste errático, para finalmente iniciar una tendencia descendente pronunciada en septiembre de 2012, y tocar un mínimo nivel de poco más de quince dólares a finales de 2014. Aunque hasta el 2012 el precio de la plata tuvo una fuerte correlación con el precio del oro, después de noviembre del 2012 parece haber disminuido, aunque la tendencia general ha sido bastante similar. 


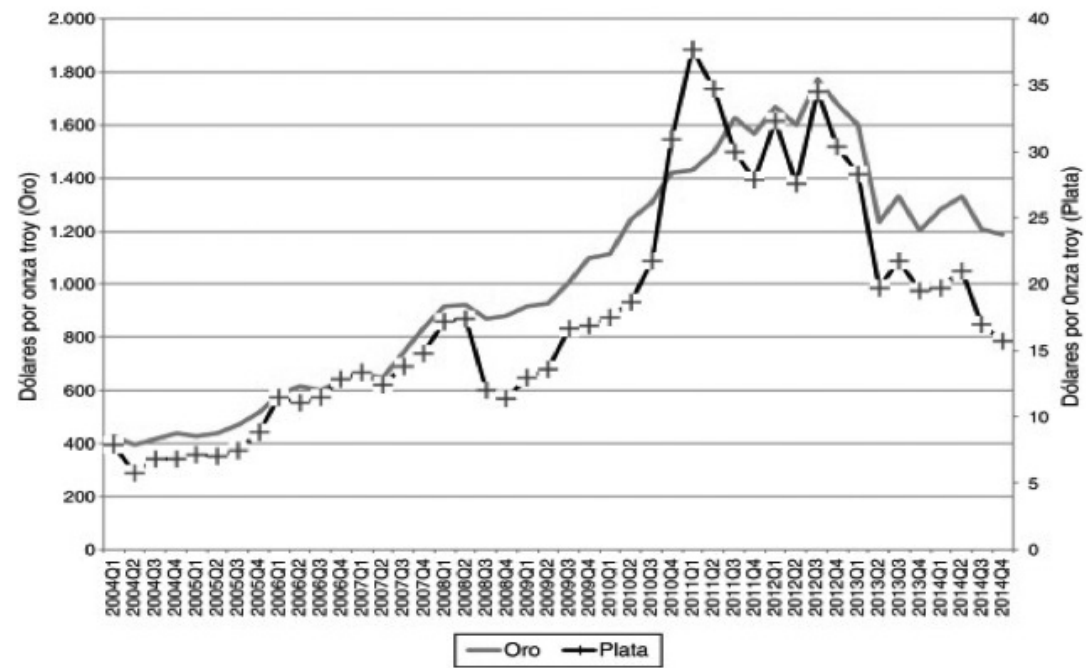

Figura 1. Precio trimestral del oro y de la plata marzo 2004 - diciembre 2014 Fuente: Bloomberg.

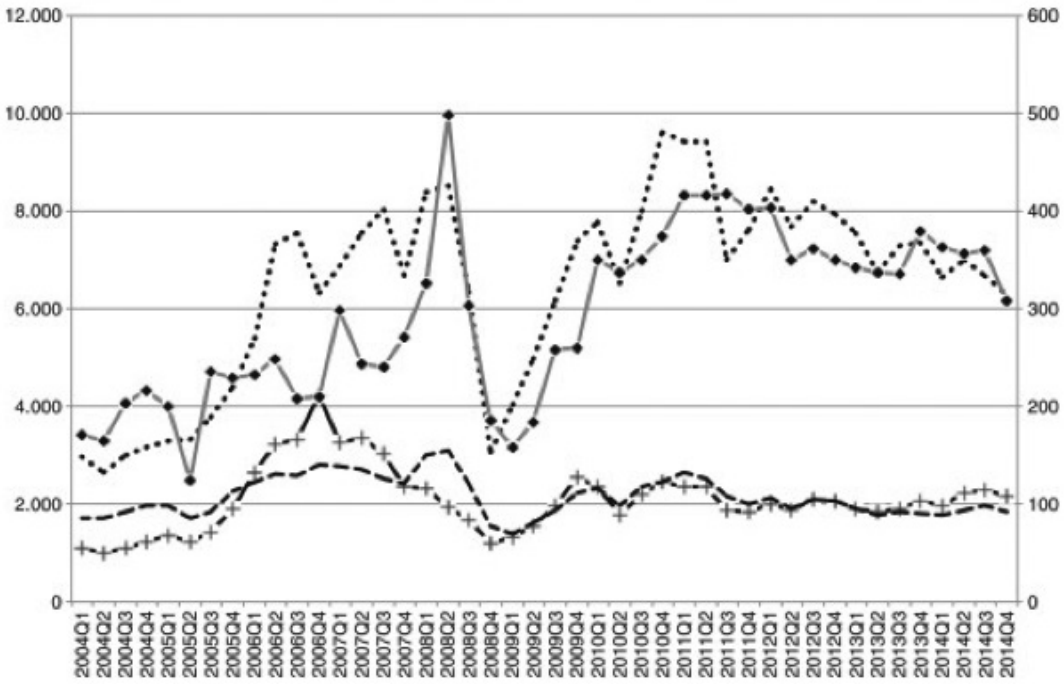

-... Cobre $\longrightarrow$ Cinc -- -Aluminio $\rightarrow$ acero

Figura 2. Precio trimestral del acero, aluminio, cobre y cinc marzo 2000 - diciembre 2014

Fuente: Bloomberg 
En su conjunto, los minerales que sirven como insumo en procesos industriales y el acero observan un comportamiento influido principalmente por el ciclo económico, aunque en cada caso existen especificidades y diferencias por el distinto grado de concentración industrial, el cambio tecnológico, las condiciones particulares de diferentes regiones productivas, etc. Aún cuando el aluminio y el acero requieren de cierto grado de transformación industrial, en realidad se trata de productos commodities, cuyo precio sigue muy de cerca el del resto de los minerales industriales.

El cobre es una materia prima industrial, cuyo precio principalmente se vio impulsado a la alza durante la primera mitad del periodo analizado por la importante demanda de la economía de China y otros países del sudeste asiático pero que, al llegar la Crisis Financiera y la desaceleración económica subsecuente, registró una baja significativa y alcanzó su nivel más bajo en diciembre de 2008, como puede verse en la Figura 2. Una vez que la economía mundial y la demanda de cobre comienzan a recuperarse, su precio alcanzó niveles superiores a los máximos del 2008 pero antes de Noviembre del 2012 inicia una nueva tendencia a la baja, consistente con la desaceleración de la economía china, la cual se mantiene hasta el final de la muestra.

El precio del zinc observó un comportamiento algo diferente con respecto al resto de los metales preciosos (o al cobre), más acorde con el uso que le da la industria. El precio se mantuvo en niveles razonablemente estables durante los primeros tres años del siglo, pero a partir de 2004 comienza a aumentar y a finales del 2006 alcanzó un máximo. A partir de ese momento inició una marcada disminución hasta fines del año 2008. Con la posterior recuperación gradual de la economía, el precio se recuperó para estabilizarse alrededor de niveles de 2000 dólares por unidad de medida.

Tabla 2.

Estadística descriptiva de los precios de los productos mineros

\begin{tabular}{lllllll}
\hline & Plata & Cobre & Cinc & Aluminio & Oro & Acero \\
\hline Media & 14.33 & 5193.47 & 1777.34 & 1963.77 & 827.91 & 241.94 \\
Desv- est. & 9.35 & 2677.18 & 784.19 & 468.52 & 474.68 & 114.99 \\
Coef. var. & 1.532838 & 1.939901 & 2.266464 & 4.191441 & 1.744125 & 2.104094 \\
\hline
\end{tabular}

Fuente: cálculos propios con datos de Standard \& Poors' Capital Markets Data Base.

En cuanto al aluminio, también siguió una tendencia básicamente relacionada con el ciclo de la economía mundial, pero con movimientos menos extremos. Entre 2000 y 2008 registró un crecimiento de aproximadamente $100 \%$, para después desplomarse en unos cuantos meses por debajo del precio inicial. En tal sentido, siguió un patrón muy similar al resto de las materias primas industriales en respuesta a la aguda desaceleración de la economía mundial asociada a la Crisis Financiera. Con el crecimiento de la economía mundial tuvo una recuperación marcada pero nunca a niveles anteriores a la crisis y una ligera baja en los últimos años.

El precio del acero para la muestra un incremento importante, del $400 \%$, entre su nivel al inicio de periodo y el máximo alcanzado durante al igual que todas las materias primas en este caso del $500 \%$, pero al inicio de la crisis mundial se marca una baja que llega a niveles de los que tenía en el 2004. La recuperación fue marcada y de casi el 100\% hasta antes del 2010 pero 
nunca llego a niveles del 2008 y al final del periodo se marca una baja constante resultado de la baja en la demanda mundial.

Debido a las diferencias de unidad de medida utilizadas para dar a conocer las cotizaciones de los distintos commodities, es difícil comparar directamente sus precios, pero es posible utilizar el coeficiente de variación de su comportamiento durante el periodo para establecer cuáles precios experimentaron una mayor volatilidad durante el periodo. En la Tabla 2 se observa que el aluminio experimentó la mayor volatilidad relativa a su precio medio, en tanto que la plata, el oro y el cobre tuvieron un comportamiento significativamente más estable.

\section{Las empresas mineras y metalúrgicas de la muestra}

La muestra de empresas mineras y metalúrgicas incluidas en este estudio representa una fracción muy limitada del universo de empresas del mismo giro en los países de Latinoamérica. La actividad minera en la región es muy importante y el número de empresas que se dedican a dicha actividad es enorme, pero la gran mayoría no son empresas bursátiles y, por esa razón es muy difícil obtener su información financiera. En total, se estudiaron catorce empresas mineras y metalúrgicas que cotizan sus acciones en las bolsas de valores de Argentina, Brasil, México y Perú, durante el periodo que comprende desde el primer trimestre de 2004 hasta el cuarto trimestre de 2014. La información extraída de los estados financieros incluyó el pasivo total, las ventas totales y la utilidad neta. Asimismo, se utilizó el precio de las acciones en el mercado al cierre de cada trimestre y el número de acciones en circulación para determinar el valor de capitalización. La razón de apalancamiento se calculó como el cociente entre el pasivo total y el valor de capitalización de las acciones en el mercado, en tanto que la razón de utilidad neta a ventas permitió contar con una medida de rentabilidad, acorde con la teoría de la estructura de capital. Los datos correspondientes a los estados financieros de las empresas fueron obtenidos de la base de datos Capital IQ, en tanto que los precios de cierre de trimestre para cada acción provienen de Bloomberg. De las catorce empresas de la muestra (ver Tabla 3), una produce aluminio, cinco producen acero, y el resto son principalmente extractivas. Por su distribución geográfica, una se ubica en Argentina, tres en Brasil, dos en México y siete en Perú.

Tabla 3.

Empresas mineras incluidas en la muestra.

\begin{tabular}{lllll}
\hline Ticker & Nombre & Producto & País & Indice \\
\hline ALUA & Aluar Aluminio Argentino S.A.I.C. & Aluminio & Argentina & MERVAL \\
ERAR & Ternium Sidecar & Acero & Argentina & MERVAL \\
CSNA3 & Companhia Siderúrgica Nacional & Acero & Brasil & BOVESPA \\
GGBR4 & Gerdau S.A. & Acero & Brasil & BOVESPA \\
GOAU4 & Metalúrgica Gerdau S.A. & Acero & Brasil & BOVESPA \\
GMEXICO B & Grupo México & Metales diversos y minería & México & IPC \\
PE\&OLES & Industrias Peñoles & Metales preciosos y otros minerales & México & IPC \\
BVN & Compañía de Minas Buenaventura & Oro & Perú & IGBVL \\
ATACOBC1 & Compañía Minera Atacocha & Metales diversos y minería & Perú & IGBVL \\
MILPOC1 & Compañía Minera Milpo & Metales diversos y minería & Perú & IGBVL \\
CORAREI1 & Corporación Aceros Arequipa & Acero & Perú & IGBVL \\
SIDERC1 & Empresa Siderúrgica del Perú & Acero & Perú & IGBVL \\
MINSURI1 & Minsur & Metales diversos y minería & Perú & IGBVL \\
VOLCABC1 & Volcán Compañía Minera & Metales diversos y minería & Perú & IGBVL \\
\hline
\end{tabular}

Fuente: Capital IQ. 


\section{Descripción de la metodología y de los resultados}

El objetivo de este trabajo consiste en determinar si el precio internacional de los minerales influye en los niveles de apalancamiento financiero, por lo cual el enfoque analítico elegido para modelar la relación fue un Análisis de Regresión Longitudinal (series de tiempo combinadas con observaciones de corte transversal), dado que la base de datos consiste de cuarenta trimestres de observaciones para catorce empresas.

Un análisis de series de tiempo para cada empresa habría enfrentado el obstáculo de contar muy pocas observaciones por empresa para lograr un ajuste satisfactorio. Puesto que la información de los estados financieros es trimestral, aún cuando los precios de los metales pueden obtenerse con periodicidad diaria (o, incluso, intradía), la información financiera de las empresas solamente está disponible con la frecuencia mencionada. Al combinar la información de todas las empresas en un Análisis de Datos en Panel, es posible subsanar el problema del tamaño de la muestra, dado que se utiliza la base de datos completa, y se logra contar con más grados de libertad para las estimaciones.

En una primera fase del análisis, se detectaron altas correlaciones entre los precios de los cuatro productos minerales y dos productos metalúrgicos considerados como posibles variables explicativas de la estructura de capital durante el periodo analizado. No es difícil explicar este fenómeno, ya que es conocida la sensibilidad de los precios de los mismos al ciclo económico mundial. Aunque efectivamente hay diferencias en su naturaleza (dos son metales preciosos, otros dos son metales industriales y los últimos dos son productos metalúrgicos), la demanda mundial está muy relacionada con la alza y baja de la actividad económica, en tanto que la oferta es fundamentalmente estable, y con variaciones muy graduales en el tiempo ${ }^{10}$.

\section{El análisis de factores}

De haber incluido los precios de cada uno de los metales incluidos en este análisis como variables explicativas, se habría producido un problema de multicolinealidad; por esa razón, se optó por utilizar el método de Análisis de Factores mediante Componentes Principales, a efecto de combinar la información contenida en las series originales de los precios de los minerales, en factores (factor scores).

El Análisis de Factores puede describirse como una técnica estadística que se utiliza para: (1) reducir el número de variables y (2) detectar la estructura de las relaciones entre las variables originales, con lo que se hace posible clasificarlas.

Para comprender mejor la manera como funciona esta técnica, a continuación se describen los pasos esenciales a seguir en su estimación. En primer lugar, se parte de un conjunto $P$ de variables aleatorias observables $\boldsymbol{X}=\left(X_{1}, \ldots, X_{p}\right)$ con medias $\boldsymbol{\mu}=\left(\boldsymbol{\mu}_{1}, \ldots, \boldsymbol{\mu}_{p}\right)$. Tenemos entonces:

$$
\boldsymbol{X}_{i}-\mu_{i}=\mathrm{L}_{i} \mathrm{~F}+\varepsilon_{\mathrm{i},} i=1,2, \ldots, p
$$

Es conveniente introducir un vector $\boldsymbol{\mu}$ de dimensión $p \times 1$, que contiene las medias de las variables originales, $\mathbf{L}$ es una matriz de coeficientes de dimensión p x m, $\mathbf{F}=\left(F_{l}, \ldots, F_{m}\right)$, es un

${ }^{10}$ Los cambios tecnológicos o la incorporación de nuevos productores al mercado mundial son fenómenos que se presentan de manera muy pausada en la industria minero metalúrgica. 
factor de variables estandarizadas no observables de dimensión m x 1, conocido como "factores comunes", y $\boldsymbol{\varepsilon}=\left(\boldsymbol{\varepsilon}_{1, \ldots .} \boldsymbol{\varepsilon}_{p)}\right.$ es un vector de errores.

El modelo expresa las p variables observables $\boldsymbol{X}-\boldsymbol{\mu}$ en términos de $m$ factores comunes

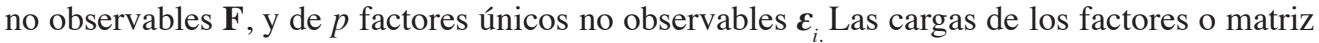
de patrón $\mathbf{L}$ vincula los factores comunes no observados a los datos observados. Así, la fila $j$ de L representa las cargas (loadings) de la variable j-esima de los factores comunes. Alternativamente, el renglón puede considerarse como los coeficientes de los factores comunes de la variable j-ésima.

Hay alguna restricciones adicionales que es necesario imponer a efecto de que $\mathrm{E}\left(F_{i}\right)=0$, $\operatorname{Var}\left(F_{i}\right)=1, \mathrm{E}(\boldsymbol{\varepsilon})=0, \operatorname{Cov}\left(\varepsilon_{i} \varepsilon_{j}\right)=0, \mathrm{E}\left(\mathbf{F F}^{\prime}\right)=\Phi, \mathrm{y} \mathrm{E}\left(\boldsymbol{\varepsilon} \varepsilon^{\prime}\right)=\Psi$, donde $\Psi$ es una matriz diagonal de varianzas únicas. Dados los supuestos anteriores, es posible derivar la relación fundamental de las varianzas del Análisis de Factores. La matriz de varianzas de las variables observadas está expresada como:

$$
\mathrm{E}\left[(\boldsymbol{X}-\boldsymbol{\mu})(\boldsymbol{X}-\boldsymbol{\mu})^{\prime}\right]=\left[(\mathbf{L F}+\varepsilon)(\mathbf{L F}+\varepsilon)^{\prime}\right]=\mathbf{L} \Phi \mathbf{L}^{\prime}+\Psi .
$$

El objetivo principal del análisis de factores (o análisis factorial) es modelar las $p(p+1) / 2$ varianzas observadas y covarianzas de $\boldsymbol{X}$ como funciones de los $p m$ pesos de factores en $\mathbf{L}$ y $p$ varianzas específicas en $\Psi .^{11}$

En la Tabla 4, se presentan los resultados del Análisis de Factores para los precios de los metales estudiados en este trabajo. En una primera salida se muestran los pesos de los dos factores extraídos, y los niveles de "comunalidad" (la proporción de la varianza de las variables originales explicada mediante los factores extraídos) y "unicidad" (la proporción de la varianza no explicada). La comunalidad permite corroborar que la técnica de Factores es capaz de capturar una proporción elevada de la varianza total original, cercana o mayor al $80 \%$, excepto en el caso del zinc (que explica solamente el 69\%).

El algoritmo utilizado para la extracción de los factores fue el método de Componentes Principales. La extracción de los pesos de los factores resultó muy interesante al asignar en el primer factor una ponderación elevada al acero, cobre, oro y la plata, en tanto que los pesos correspondientes al zinc y al aluminio fueron relativamente bajos. Sin embargo, en el segundo factor, los pesos correspondientes a estos dos últimos aumentaron significativamente. La proporción de la varianza explicada por el modelo alcanza 69\% para el primer factor y $31 \%$ para el segundo factor. Con base en las cargas de los factores no rotados se estimaron los valores de los factores (Factor Scores) a fin de reducir la dimensionalidad del modelo de seis variables originales a solamente dos. Los valores de los factores se utilizan en la siguiente etapa del análisis como insumo.

Para lograr una interpretación más clara del sentido de los resultados del análisis de factores, se procedió a realizar una rotación de factores ${ }^{12}$ mediante el método varimax. Esta vez, los pesos del primer factor indican claramente una relación fuerte, mayor o igual a $95 \%$, con el oro y la plata, y en menor medida con el cobre y el acero $76 \%$ y $85 \%$, respectivamente. Gracias a la rotación, y se logra una decantación aún mayor respecto al aluminio y al zinc en el primer factor y, el segundo factor destaca claramente la ponderación del aluminio y el zinc. La Tabla 4 se presentan las salidas de ambos métodos de estimación.

\footnotetext{
${ }^{11}$ Chapter 39, Factor Analysis, EViews 7 User Guide II, pp. 736-737, basado en Johnston y Wichtern (1992).

${ }^{12} \mathrm{La}$ "rotación de factores" es una técnica que consiste en modificar las coordenadas de los ejes originales para lograr una interpretación más intuitiva de las salidas del Análisis de Factores (ver, por ejemplo, Chapter 39, Factor Analysis, EViews 7 User Guide II.
} 
Tabla 4

Cargas de los factores originales y de los factores rotados con el método Varimax

Método de componentes principales

Análisis de covarianza: correlación ordinaria

Muestra: 2004Q1 2014Q4 Observaciones incluidas: 616

Comunalidades previas: correlación cuadrada múltiple

\section{Factores sin rotación}

F1

Acero

Aluminio

Cobre

Plata

Oro

Cinc

Factor

F1

F2

Total

2. Factores rotados

Acero

Aluminio

0.849893

Cobre

0.054106

Plata

0.757483

Oro

0.949198

0.97732

0.06917

Cinc
F2

$-0.165475$

0.794907

0.209137

$-0.337623$

$-0.505347$

0.712229

Acumulada

3.552046

5.131679

5.131679

F2

0.238478

0.915016

0.61096

0.09562

$-0.077751$

0.830152

\section{Comunalidad}

0.77919

0.840181

0.947052

0.91012

0.961199

0.693936

Diferencia

1.972414

---
Unicidad

0.22081

0.159819

0.052948

0.08988

0.038801

0.306064

Fuente: cálculos propios con EViews 9.

Para lograr una interpretación más clara del sentido de los resultados del análisis de factores, se procedió a realizar una rotación de factores13 mediante el método varimax. Esta vez, los pesos del primer factor indican claramente una relación fuerte, mayor o igual a $95 \%$, con el oro y la plata, y en menor medida con el cobre y el acero $76 \%$ y $85 \%$, respectivamente. Gracias a la rotación, y se logra una decantación aún mayor respecto al aluminio y al zinc en el primer factor y, el segundo factor destaca claramente la ponderación del aluminio y el zinc. La Tabla 4 se presentan las salidas de ambos métodos de estimación.

\footnotetext{
${ }^{13}$ La "rotación de factores" es una técnica que consiste en modificar las coordenadas de los ejes originales para lograr una interpretación más intuitiva de las salidas del Análisis de Factores (ver, por ejemplo, Chapter 39, Factor Analysis, EViews 7 User Guide II.
} 


\section{El análisis de regresión transversal}

En la segunda parte del análisis empírico se parte del supuesto de que la estructura de capital de las empresas minero-metalúrgicas de la muestra, estimada como la razón de Deuda Total/ Valor de Capitalización, es una variable dinámica que responde a las variaciones del precio de las acciones correspondientes. En tal virtud, aunque es irrealista asumir que los administradores financieros modifican la composición de las fuentes de financiamiento en el balance general como respuesta al comportamiento de los precios de los productos que venden inmediatamente (esto sucede, efectivamente, los precios suben o bajan de una manera más o menos prolongada, pero se verá reflejado varios trimestres después del inicio de la tendencia), debido a que el precio de las acciones refleja de manera inmediata e insesgada la información relevante para su valuación, es factible afirmar que el valor de capitalización sí responde de manera dinámica a las fluctuaciones de los precios.

Para determinar la sensibilidad en cuanto a la magnitud y dirección de la influencia de los precios de los metales sobre la estructura de capital de la empresa se define a ésta última como la variable a explicar, en tanto que los valores de los dos factores estimados mediante el Análisis de Factores, que representan el comportamiento de los precios de los seis productos minerales y metalúrgicos, junto con la razón de utilidad neta a ventas trimestrales, se incluyen como variables explicativas.

Es de esperar que la elevación del precio de los productos de estas empresas, aumenten su nivel de ingresos y utilidad, pues la estructura de costos resultante de una planta física y procesos tecnológicos poco cambiantes no afecta el resultado. Por lo tanto, el signo de los coeficientes correspondientes a los factores que representan los precios de los seis productos estudiados sea negativo. Asimismo, la relación de utilidad neta a ventas es un reflejo de la rentabilidad de mediano plazo de la empresa, pues depende de principalmente de su eficiencia, aunque dada la estabilidad relativa de los costos de producción y administración, también es sensible a los altibajos en los precios del producto.

El modelo propuesto para evaluar la relación entre las variables explicativas y la estructura de capital de las empresas puede expresarse de la manera siguiente:

(Deuda Total/Capitalización de Mercado) $_{i t}=$

$$
\alpha+\beta_{1}(\text { Factor } 1)_{i t}+\beta_{2}(\text { Factor } 2)_{i t}+\varepsilon
$$

Los resultados del modelo se presentan a continuación en la Tabla 5. Tanto el signo de las tres variables explicativas, como la significancia estadística de las variables, confirman que el modelo tiene un sustento empírico sólido. Además de la Regresión Transversal sencilla, se consideró la posibilidad de realizar un Análisis de Datos en Panel con efectos fijos, aleatorios o ambos, para permitir que el modelo tuviera la capacidad de reconocer las diferencias naturales entre empresas, productos, y regiones geográficas donde operan. Es interesante que tanto el modelo de efectos fijos como el de efectos aleatorios fueron rechazados pues no mejoraron la capacidad explicativa del modelo de manera suficiente para reemplazarlo. Para el efecto se llevaron a cabo las estimaciones correspondientes y la prueba de Efectos Fijos Redundantes, en el caso del modelo de efectos fijos (Redundant Fixed Effects Test), y la prueba de Hausman, para el modelo de efectos aleatorios. Los resultados de las estimaciones y de las pruebas correspondientes se omiten en este trabajo por razones de espacio. ${ }^{14}$

\footnotetext{
${ }^{14}$ Sin embargo, se hacen disponibles a petición explícita del interesado a los autores de contacto.
} 
Tabla 5.

Regresión transversal para el apalancamiento financiero como función de los factores de precios de los metales y de la rentabilidad de las empresas

Variable dependiente: D (deuda total/capitalización de mercado)

Método: panel mínimos cuadrados

Muestra (ajustada): 2004Q2 2014Q4

Periodos incluidos: 43

Unidades de sección cruzada incluidas: 14

Observaciones totales en el panel: 600

\begin{tabular}{|c|c|c|c|c|}
\hline Variable & Coeficiente & Error estándar & Estadístico- t & Prob. \\
\hline $\mathrm{C}$ & 0.015433 & 0.004575 & 3.372894 & 0.0008 \\
\hline $\mathrm{D}(\mathrm{F} 1)$ & -0.030876 & 0.016294 & -1.894931 & 0.0586 \\
\hline $\mathrm{D}(\mathrm{F} 2)$ & -0.038047 & 0.00699 & -5.443414 & 0 \\
\hline RENTAB & -0.047098 & 0.014585 & -3.229232 & 0.0013 \\
\hline R-cuadrada & 0.101230 & \multicolumn{2}{|c|}{ Varianza de la media depend } & 0.006231 \\
\hline R-cuadrada ajustada & 0.096713 & \multicolumn{2}{|c|}{ E.S. de la var. depend } & 0.101652 \\
\hline $\mathrm{E}, \mathrm{S}$ de regresión & 0.096612 & \multicolumn{2}{|l|}{ Criterio Akaike } & -1.829603 \\
\hline Suma resid cuadrados & 5.572275 & \multicolumn{2}{|c|}{ Criterio de Schwarz } & -1.800328 \\
\hline Verosimilitud Log & 553.7958 & \multicolumn{2}{|c|}{ Criterio de Hannan-Quinn } & -1.818208 \\
\hline Estadístico- F & 22.41368 & \multicolumn{2}{|l|}{ Durbin-Watson } & 1.806666 \\
\hline Prob (estadístico-F) & 0.00000 & & & \\
\hline
\end{tabular}

Fuente: cálculos propios con EViews 9.

\section{Conclusiones}

Los resultados econométricos sugieren que la rentabilidad de las empresas mineras y el comportamiento del precio de sus productos (commodities metalúrgicos) son variables que afectan negativamente la estructura de capital de las empresas debido al impacto que tienen sobre los flujos de efectivo de las empresas. Por lo mismo, la principal contribución de nuestro análisis consiste en aportar evidencia a favor de la Teoría del Orden Selectivo de Preferencia Dinámico en el ámbito de la muestra de empresas mineras de los países latinoamericanos estudiadas.

Sin embargo, la evidencia también puede ser de utilidad para el diseño de estrategias de cobertura contra riesgos de mercado por las empresas del sector, a fin de minimizar el impacto potencial que hasta ahora tienen las fluctuaciones de los precios de sus productos sobre su estructura de capital. Un aumento no planeado del apalancamiento de una empresa, afectada por la baja de los precios de sus productos, pone en riesgo su calificación crediticia y eleva el costo de sus fuentes de financiamiento. Los resultados también tienen interés para los administradores de portafolios, interesados en minimizar la volatilidad de sus portafolios de 
bonos (afectados por cambios en la calificación crediticia) y en el nivel de exposición al riesgo de mercado de las acciones incorporadas en sus carteras.

\section{Referencias}

Bessler, W., Drobetz, W. y Kazemieh, R. (2011). Factors affecting capital structure decisions. In Capital Structure and Corporate Financing Decisions: Theory, Evidence, and Practice. New Jersey: John Wiley. https://doi.org/10.1002/9781118266250.ch2

Booth, L., Aivazian, V., Demirguc-Kunt, A. y Maksimovic, V. (2001). Capital structures in developing countries. The Journal of Finance, LCI, (1), 87-130. https://doi.org/10.2469/dig.v31.n3.909

Frank, M. Z. y Goyal, V. K. (2003). Testing the pecking order theory of capital structure. Journal of Financial Economics, 67(2), 217-248, http://doi.org/10.1016/S0304-405X(02)00252-0.

Frank, M. Z. y Goyal, V. K. (2009). Capital structure decisions: Which factors are reliably important? Financial Management, 38(1), 1-37. http://doi.org/10.1111/j.1755-053X.2009.01026.x.

Gómez, A. P., Castro, G. Á. y Ortega, M. F. (2016). Determinants of leverage in mining companies, empirical evidence for Latin American countries. Contaduría y Administración, 61(1), 26-40. https://doi.org/10.1016/j.cya.2015.09.010

Graham, J. R. y Leary, M. T. (2011). A review of empirical capital structure research and directions for the future (April 7, 2011). Annual Review of Financial Economics, 3. https://doi.org/10.2139/ssrn.1729388

Harris, M. y Raviv, A. (1991). The theory of capital structure. The Journal of Finance, 46(1), 297-355. Disponible en: https://onlinelibrary.wiley.com/doi/full/10.1111/j.1540-6261.1991.tb03753.x Consultado : 01/06/2016.

Jensen, M. C. y Meckling, W. H. (1976). Theory of the firm: managerial behavior, agency costs and ownership structure theory of the firm: managerial behavior, agency costs and ownership structure. Journal of Financial Economics, 3(4), 305-360, http://doi.org/http://dx.doi.org/10.1016/0304-405X(76)90026 X.

Jõeveer, K. (2006). Sources of capital structure: evidence from transition countries. Bank of Estonia, Working Papers Series, Number 2/2006. https://doi.org/10.2139/ssrn.1130306

Kraus, A. y Litzenberger, R. H. (1973). A state-preference model of optimal financial leverage. The Journal of Finance, 28, 911-922. http://dx.doi.org/10.1111/j.1540-6261.1973.tb01415.x

Maquieira, C., Olavarrieta, S. y Zutta, P. (2007). Determinantes de la estructura de capital. Evidencia empírica para Chile. El Trimestre Económico LXXIV, (1), 161-193. Disponible en: http://www.redalyc.org/pdf/313/31340949004.pdf Consultado: 01/06/2016.

Medina, A., Salinas, J., Ochoa, L. y Molina, C. (2012). La estructura financiera de las empresas manufactureras colombianas, una mirada econométrica, macroeconómica y financiera. Revista Científica de América Latina, El Caribe, España y Portugal, (20), 1-28. Disponible en: http://www.scielo.org.co/pdf/pece/n20/n20a7.pdf Consultado: 01/06/2016.

Modigliani, F. y Miller, M. (1958). The cost of capital, corporation finance and the theory of investment. American Economic Review, 48(3), 261-297.https://doi.org/10.4013/base.20082.07

Disponible en: https://s3.amazonaws.com/academia.edu.documents/30867396/modiglianiandmiller1958.pdf?AWSAccessKeyId=AKIAIWOWYYGZ2Y53UL3A\&Expires=1529450306 $\&$ Signature=z9wqByp $\% 2 F 5$ stImKzsC0q2graDR3Q\%3D\&response-content-disposition=inline\%3B\%20filename\%3DThe_Cost_of_Capital_Corporation_Finance.pdf Consultado : 01/06/2016.

Modigliani, F. y Miller, M. (1963). Corporate income taxes and the cost of capital: a correction. American Economic Review, 53(3), 433-443. Disponible en: https://s3.amazonaws.com/academia.edu.documents/30927825/modiglianimiller3.pdf?AWSAccessKeyId=AKIAIWOWYYGZ2Y53UL3A\&Expires=1529450458\& Signature=WbpHR0E\%2F5cu\%2Bkned2tBpe9GPJSk\%3D\&response-content-disposition=inline\%3B\%20filename\%3DCorporate_income_taxes_and_the_cost_of_c.pdf Consultado: 01/06/2016. 
Mongrut, S., Fuenzalida, D., Pezo, G. y Teply, Z. (2010). Explorando teorías de estructura de capital en Latinoamérica. Cuadernos de Administración, 23(41), 163-184. Disponible en: http://www.scielo.org.co/pdf/cadm/v23n41/ v23n41a08.pdf Consultado: 01/06/2016.

Myers, S. C. (1977). Determinants of corporate borrowing. Journal of Financial Economics, 5(5), 147-175. https://doi.org/10.1016/0304-405x(77)90015-0

Myers, S. C. (1984). The capital structure puzzle. The Journal of Finance, 39(3), 575-592. https://doi.org/10.3386/ w1393

Myers, S. C. y Majluf, N. S. (1984). Corporate financing and investment decisions when firms have information that investors do not have. Journal of Financial Economics, 13(2), 187-221, http://doi.org/10.1016/0304405X(84)90023-0.

Paredes, A. y Flores-Ortega, M. (2012). Análisis del comportamiento de la estructura de capital de empresas mexicanas del sector siderúrgico periodo 2001-2011. ESECONOMIA, VII, 45-72. https://ideas.repec.org/a/ipn/esecon/ vviiy2012i36p45-72.html (Junio 18, 2018).

Paredes Gómez, A., Ángeles Castro, G., \& Flores Ortega, M. (2016). Determinants of leverage in mining companies, empirical evidence for Latin American countries. Contaduría y Administración, 61(1), 26-40. http://doi. org/10.1016/j.cya.2015.09.010

Shyam-Sunder, L. y Myers, S. C. (1999). Testing static tradeoff against pecking order models of capital structure. Journal of Financial Economics, 51, 219-244. https://doi.org/10.3386/w4722 